\title{
Rapid ventricular pacing for clip reconstruction of complex unruptured intracranial aneurysms: results of an interdisciplinary prospective trial
}

\author{
*Juergen Konczalla, MD, PhD, ${ }^{1}$ Johannes Platz, MD, ${ }^{1}$ Stephan Fichtlscherer, MD, PhD, ${ }^{2}$ \\ Haitham Mutlak, MD, ${ }^{3}$ Ulrich Strouhal, MD, ${ }^{3}$ and Volker Seifert, MD, PhD ${ }^{1}$
}

Departments of ${ }^{1}$ Neurosurgery and ${ }^{2}$ Cardiology, University Hospital Frankfurt, Goethe University; and ${ }^{3}$ Department of Anesthesiology, Intensive Care Medicine and Pain Therapy, University Hospital Frankfurt, Goethe University, Frankfurt, Germany

OBJECTIVE To date, treatment of complex unruptured intracranial aneurysms (UIAs) remains challenging. Therefore, advanced techniques are required to achieve an optimal result in treating these patients safely. In this study, the safety and efficacy of rapid ventricular pacing (RVP) to facilitate microsurgical clip reconstruction was investigated prospectively in a joined neurosurgery, anesthesiology, and cardiology study.

METHODS Patients with complex UIAs were prospectively enrolled. Both the safety and efficacy of RVP were evaluated by recording cardiovascular events and outcomes of patients as well as the amount of aneurysm occlusion after the surgical clip reconstruction procedure. A questionnaire was used to evaluate aneurysm preparation and clip application under RVP.

RESULTS Twenty patients (mean age 51.6 years, range $28-66$ years) were included in this study. Electrode positioning was easy in $19(95 \%)$ of 20 patients, and removal of electrodes was easily accomplished in all patients (100\%). No complications associated with the placement of the pacing electrodes occurred, such as cardiac perforation or cardiac tamponade. RVP was applied in 16 patients. The mean aneurysm size was $11.1 \pm 5.5 \mathrm{~mm}$ (range $6-30 \mathrm{~mm}$ ). RVP proved to be a very helpful tool in aneurysm preparation and clip application in $15(94 \%)$ of 16 patients. RVP was used for a mean duration of $60 \pm 25$ seconds, a mean heart rate of $173 \pm 23$ bpm (range 150-210 bpm), and a reduction of mean arterial pressure to $35-55 \mathrm{~mm} \mathrm{Hg}$. RVP leads to softening of the aneurysm sac facilitating its mobilization, clip application, and closure of the clip blades. In 2 patients, cardiac events were documented that resolved without permanent sequelae in both. In every patient with successful RVP $(n=14)$ a total or near-total aneurysm occlusion was documented. In the 1 patient in whom the second RVP failed due to pacemaker electrode dislocation, additional temporary clipping was required to secure the aneurysm, but was not as sufficient as RVP. This led to an incomplete clipping of the aneurysm and finally a remnant on postoperative digital subtraction angiography. A pacemaker lead dislocation occurred in $3(19 \%)$ of 16 patients, but intraoperative repositioning requires less than 20 seconds. Outcome was favorable in all patients according to the modified Rankin Scale.

CONCLUSIONS To the best of the authors' knowledge this is the first prospective interdisciplinary study of RVP use in patients with UIAs. RVP is an elegant technique that facilitates clip reconstruction in complex UIAs. The safety of the procedure is good. However, because this procedure requires extensive preoperative cardiological workup of the patient and an experienced neurosurgery and neuroanesthesiology team with much cerebrovascular expertise, actually it remains reserved for selected elective cases and highly specialized centers.

Clinical trial registration no.: NCT02766972 (clinicaltrials.gov)

https://thejns.org/doi/abs/10.3171/2016.11.JNS161420

KEY WORDS rapid ventricular pacing; large, giant, or complex aneurysm; clipping; surgical; vascular disorders

ABBREVIATIONS ACOA = anterior communicating artery; $C K=$ creatine kinase; $E C G=$ electrocardiogram; $D S A=$ digital subtraction angiography; ICA = internal carotid artery; ICG = indocyanine green; ICU = intensive care unit; IOM = intraoperative monitoring; MAP = mean arterial pressure; MCA = middle cerebral artery; MEP = motor evoked potential; $\mathrm{mRS}=$ modified Rankin Scale; $\mathrm{PCoA}=$ posterior communicating artery; RVP = rapid ventricular pacing; $\mathrm{SAH}=$ subarachnoid hemorrhage; $\mathrm{SSEP}=$ somatosensory evoked potential; TTE = transthoracic echocardiography; UIA = unruptured intracranial aneurysm.

ACCOMPANYING EDITORIAL See pp 1737-1740. DOI: 10.3171/2017.1.JNS162923.

SUBMITTED June 3, 2016. ACCEPTED November 17, 2016.

INCLUDE WHEN CITING Published online August 18, 2017; DOI: 10.3171/2016.11.JNS161420.

* Drs. Konczalla and Platz contributed equally to this work. 
$\mathrm{C}$ URRENTLY, most aneurysms can be treated by primary clip reconstruction or by endovascular coil embolization (with or without stents), or with newer devices such as flow diverters or a woven endobridge (WEB) device (Sequent Medical). However, morbidity, mortality, and mid- to long-term occlusion rates of the newer endovascular devices have not been as good as expected. ${ }^{7,8,15,32}$ With increasing size and complexity of the aneurysm (wide neck, calcifications, incorporation of branching vessels), the rate of fully occluded aneurysms on postoperative digital subtraction angiography (DSA) decreases while the risk of procedure-related morbidity and mortality increases. ${ }^{10,34}$

However, the number of complex and large or giant unruptured intracranial aneurysms (UIAs) is rising and treatment remains challenging to date. Different tools and techniques can be used surgically in complex aneurysms, such as temporary clipping, adenosine-induced flow arrest, suction decompression, or deep hypothermia with cardiac standstill., ${ }^{4,14,43}$ Finally, bypass techniques and parent artery occlusion could be the last remaining choices. ${ }^{20,27,31}$ All of these tools and techniques have advantages and disadvantages, which will be discussed later. Recently, a small series and 3 case reports described the successful use of rapid ventricular pacing (RVP) during aneurysm surgery after subarachnoid hemorrhage (SAH). ${ }^{21,30,38,45}$

RVP for flow reduction appears to be a reasonable tool for complex cases..$^{37,38,45}$ In contrast to adenosine, RVP allows one to control the start time, the length of pacing, and the induced flow/pressure reduction under controlled conditions. ${ }^{36,38}$ Furthermore, the brain parenchyma is still perfused. To date, there are only limited data available for this technique. In this prospective interdisciplinary trial, we focused on patient safety, simplicity of use, efficacy of flow reduction, and improvement of clip application. In this paper we present the data for our first 20 patients.

\section{Methods}

The study was approved by the local ethics committee of University Hospital Frankfurt. All patients gave their written informed consent to participate in the study. Only patients with a complex, large, or giant aneurysm were screened for possible trial inclusion.

Inclusion criteria for the study were: 1) age $\geq 18$ years, 2) complex UIA intended to be treated by microsurgical clip reconstruction, and 3) application of RVP intraoperatively. Because this study was conducted as a pivotal trial, we only included patients with no obvious structural heart disease or conductance abnormalities, obtained by case history, surface electrocardiogram (ECG), treadmill ECG, and transthoracic echocardiography (TTE). This cardiac workup (surface ECG, treadmill ECG, and TTE) takes less than 3 hours. Furthermore, patients were excluded if they had an increased general periprocedural risk as judged by the anesthesiologist.

\section{Preoperative Workup}

After interdisciplinary consensus on aneurysm treatment, patients selected to undergo microsurgical clipping were screened to determine whether the use of RVP would be considered to be helpful during surgery due to aneurysm complexity, size, and/or location (Fig. 1). ${ }^{23,28,46}$

All patients considered for RVP during surgery were extensively evaluated by a cardiologist (S.F.) prior to the procedure, including a TTE to detect abnormal wall movements, valvular heart disease, or intraventricular thrombi. Furthermore, a stress ECG was performed. One patient was excluded because the treadmill ECG showed ST elevations and the patient required cardiac stents. Before surgery, 2 neuroanesthesiologists (H.M. and U.S.) consulted with the patient and evaluated the periprocedural risk based on the cardiology workup and the general condition of the patient.

\section{Anesthesia Procedure, Probe Placement, and RVP Testing}

Patients received preoperative oral premedication $(7.5$ $\mathrm{mg}$ of midazolam). Before anesthesia induction a peripheral intravenous access line and a radial artery catheter for continuously monitoring arterial blood pressure were placed. Anesthesia was induced with sufentanil, propofol, and rocuronium and maintained with continuous application of remifentanil and propofol according to the intraoperative needs. After insertion of a central venous catheter (9.5-Fr diameter, 5 lumens, $20 \mathrm{~cm}$ long) for central venous pressure monitoring and drug administration, a vascular introducer sheath with a large infusion port (Arrow Flex FEP Sheath, 6-Fr diameter, 10-cm length) was placed into the subclavian vein. Standard monitoring included pulse oximetry, ECG, invasive blood pressure monitoring, capnography, and esophageal and bladder temperature. A 5-Fr balloon temporary pacing catheter (Arrow, Vascostim) was introduced through the vascular introducer in the subclavian vein into the right ventricle. Correct positioning of the pacing electrode was confirmed via continuous ECG monitoring under pacer stimulation with a low pacing threshold. External defibrillation pads were placed on the chest wall.

\section{Surgical Procedure and Intraoperative Monitoring}

Clip placement as well as complex clip reconstruction techniques were performed by the senior author (V.S.) in a standardized microsurgical procedure, opening the sylvian fissure and basal cisterns. All procedures were conducted using intraoperative monitoring (IOM) of somatosensory evoked potentials (SSEPs) and motor evoked potentials (MEPs). ${ }^{42}$ The patients were kept normovolemic with a mean arterial pressure (MAP) of 70-100 $\mathrm{mm} \mathrm{Hg}$. Additionally, mild hypothermia $\left(35^{\circ} \mathrm{C}\right)$ and burst suppression were induced before aneurysm clipping. A pterional craniotomy was used as the standard approach for all aneurysms. Intraoperatively, indocyanine green (ICG) video angiography was used to verify aneurysm occlusion as well as parent vessel perfusion after each clip placement. ${ }^{35}$ Initially, we tried to clip the aneurysm without additional techniques, but if this was not possible, the next step was to use RVP. However, in patients without sufficient flow reduction by RVP, other techniques were allowed, such as temporary clipping or induced hypotension. We differentiated between whether insufficient flow reduction was due to pacemaker dislocation or due to RVP by itself; this 


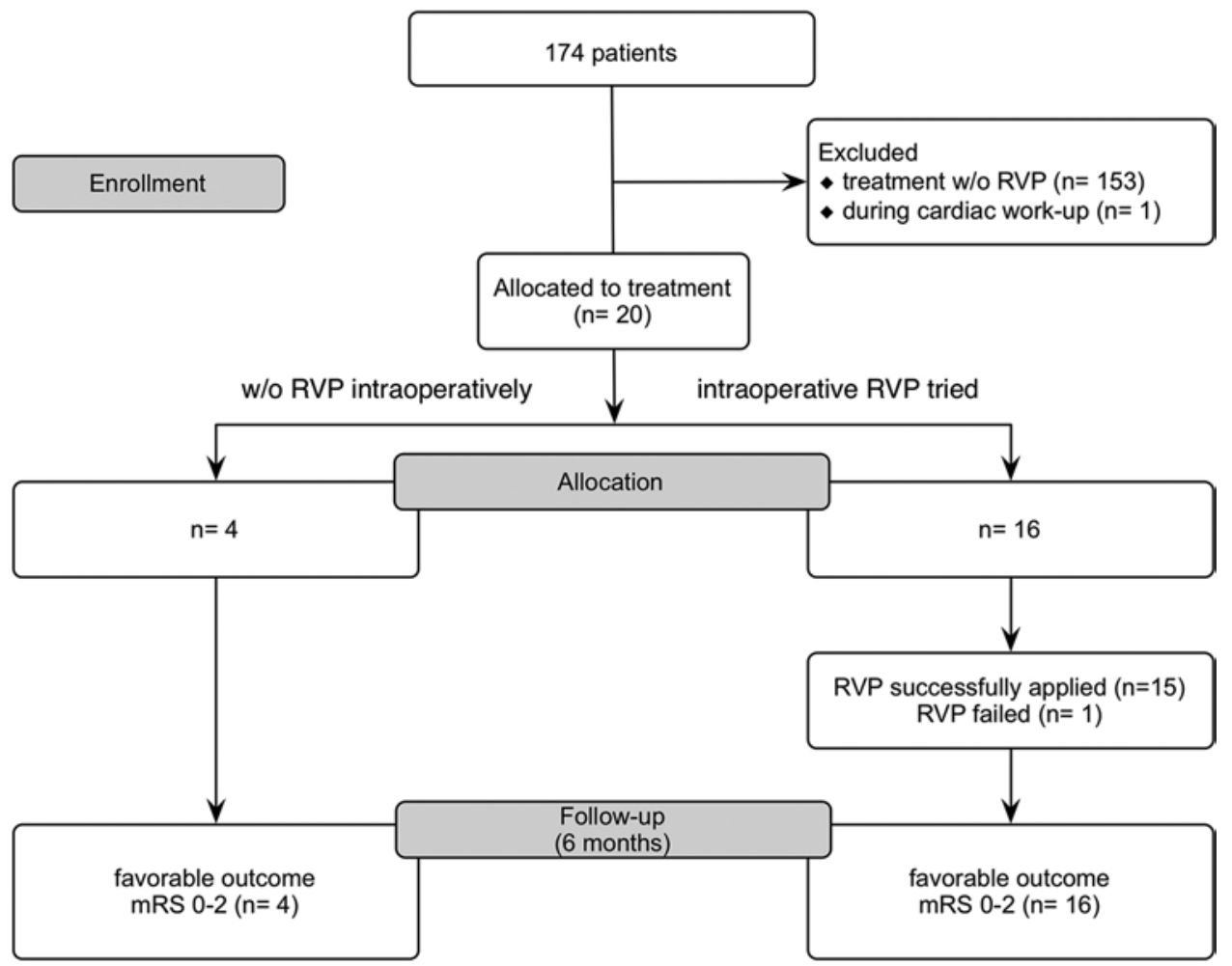

FIG. 1. Flow diagram. During the study period, of 174 treated patients, 21 were selected for possible intraoperative RVP. One patient was excluded during preoperative workup because of ST elevations during treadmill ECG. Intraoperatively, RVP was not required to clip the aneurysm in 4 patients. RVP failed in 1 patient completely. Outcome after 6 months was favorable in every patient.

could be easily identified by a change of the QRS complex under sufficient RVP (Fig. 2).

Initially, RVP was started at a rate of $150 \mathrm{bpm}$ and gradually increased to approximately $200 \mathrm{bpm}$ until the effect on blood pressure was registered. The primary goal was to "titrate" the MAP to or below $50 \mathrm{~mm} \mathrm{Hg}$, depending on the surgeon's preference (e.g., intraaneurysmal pressure reduction). A pulsatile flow in the arterial wave form was demanded, to maintain a minimum cardiac output during the procedure.

\section{Cardiac and Postoperative Workup}

After surgery, all patients were admitted to the neurosurgical intensive care unit (ICU). Cardiac enzymes were

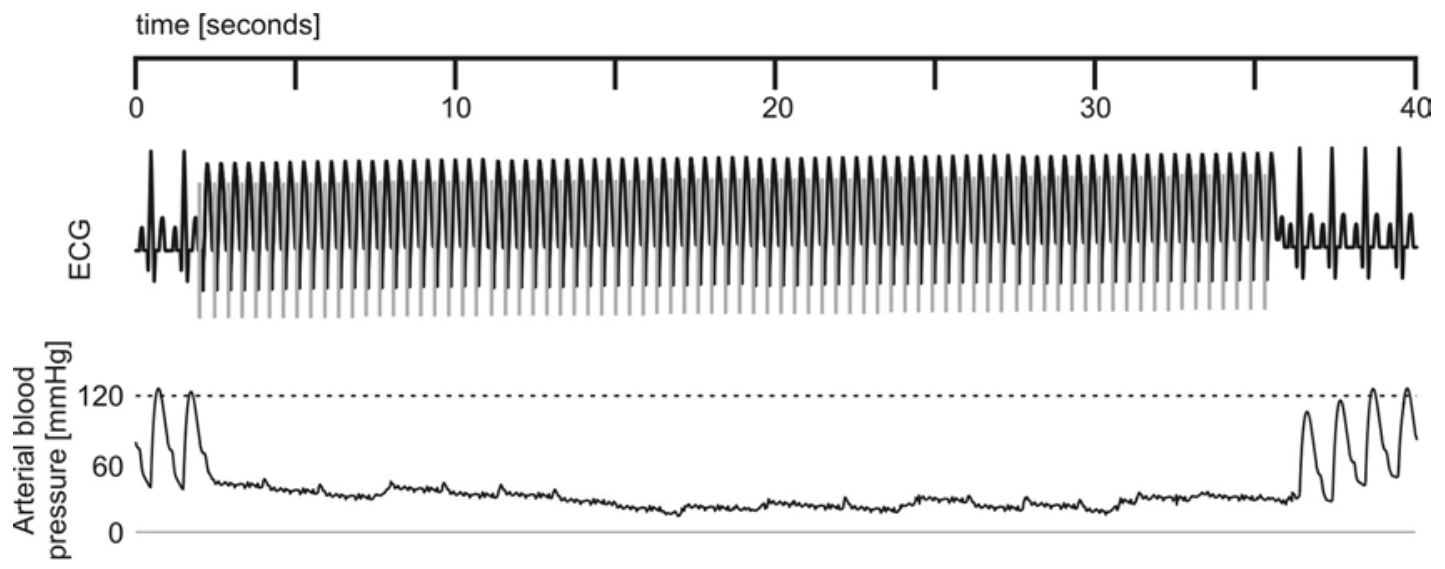

FIG. 2. Heart rhythm (ECG, upper) and blood pressure (lower) during the course of 1 RVP in 1 patient. After 2 normal QRS complexes, RVP was started for about 35 seconds. During this period the arterial blood pressure drops immediately (lower) and returns to normal values within seconds, without vasoactive agents. 
TABLE 1. Characteristics of the patients and aneurysms included in this study

\begin{tabular}{|c|c|c|c|c|c|c|}
\hline $\begin{array}{l}\text { Case } \\
\text { No. }\end{array}$ & $\begin{array}{c}\text { Age (yrs), } \\
\text { Sex }\end{array}$ & $\begin{array}{l}\text { Aneurysm } \\
\text { Site }\end{array}$ & $\begin{array}{l}\text { Aneurysm } \\
\text { Size }(\mathrm{mm})\end{array}$ & Comment & Occlusion & $\begin{array}{l}\text { No. of } \\
\text { Clips }\end{array}$ \\
\hline 1 & $66, M$ & $\mathrm{ACoA}$ & 18 & Wide neck & Complete & 2 \\
\hline 2 & $58, F$ & MCA & 9 & Wide calcified neck, including $\mathrm{M}_{2}$ origins & Residual neck & 2 \\
\hline 3 & $54, \mathrm{~F}$ & $\mathrm{PCoA}$ & 9 & Wide calcified neck, including PCoA and AChA origins, dysplastic & Residual neck & 2 \\
\hline $4^{*}$ & $28, \mathrm{M}$ & MCA & 10 & $\begin{array}{l}\text { Recanalized large MCA aneurysm w/ coil protrusion into neck \& } \\
\text { emerging perforator }\end{array}$ & Residual neck & 2 \\
\hline 5 & $57, \mathrm{~F}$ & $\mathrm{ACOA}$ & 9 & Wide calcified neck, including $A_{2}$ origins & Complete & 4 \\
\hline $6^{*}$ & $52, \mathrm{~F}$ & MCA & 8 & Wide calcified neck & & 4 \\
\hline 7 & $45, \mathrm{~F}$ & ICA (paraclinoid) & 6 & Paraclinoid aneurysm & Residual neck & 1 \\
\hline $8^{*}$ & $47, \mathrm{~F}$ & MCA & 8 & Wide neck, including $\mathrm{M}_{2}$ origins & & 2 \\
\hline 9 & $46, \mathrm{~F}$ & MCA & 10 & Wide calcified neck & Complete & 1 \\
\hline $10^{*}$ & $56, \mathrm{~F}$ & MCA & 8 & Wide neck heavily calcified, dysplastic part; fusiform $\mathrm{M}_{1}$ & $\mathrm{NA} \dagger$ & 0 \\
\hline 11 & $37, \mathrm{~F}$ & MCA & 8 & Wide neck, including $\mathrm{M}_{2}$ origins & Complete & 1 \\
\hline 12 & $57, \mathrm{~F}$ & ICA (bifurcation) & 7 & Prior endovascular treatment failed & Complete & 1 \\
\hline 13 & $62, \mathrm{~F}$ & MCA & 17 & Wide neck, including $\mathrm{M}_{2}$ origins & Complete & 4 \\
\hline 14 & $55, \mathrm{M}$ & PCoA & 14 & Wide neck, including PCoA origin \& posterior wall of ICA & Nonef & 0 \\
\hline 15 & $48, M$ & MCA & 30 & Dysplastic calcified neck including $\mathrm{M}_{2}$ origins & Complete & 9 \\
\hline 16 & $48, F$ & ICA (paraclinoid) & 15 & Large paraclinoid aneurysm including supraopthalmic origin & Complete & 2 \\
\hline 17 & $56, F$ & MCA & 9 & Wide neck, including $\mathrm{M}_{2}$ origins & Residual neck & 5 \\
\hline 18 & $63, \mathrm{~F}$ & $\mathrm{ACoA}$ & 9 & Wide neck, including $A_{2}$ origins, $A_{2}$ adherent to aneurysmal sac & Residual sac & 1 \\
\hline 19 & $55, \mathrm{~F}$ & MCA & 10 & Wide neck, including $\mathrm{M}_{2}$ origins & Residual neck & 4 \\
\hline 20 & $43, M$ & MCA & 8 & Wide neck, including $\mathrm{M}_{2}$ origins & Residual neck & 2 \\
\hline
\end{tabular}

AChA = anterior choroidal artery; NA = not available.

* RVP not applied intraoperatively.

† Not available, because aneurysm was not clipped.

‡ RVP not possible intraoperatively due to technical failure, most likely displacement of the pacemaker electrode. Without RVP, clipping of the aneurysm could not be achieved.

controlled preoperatively, at ICU admission and the day after the procedure. All patients were monitored with continuous ECG for 24 hours. On Day 7, aneurysm occlusion was assessed by control DSA. Patient outcome was assessed using the modified Rankin Scale (mRS) during an outpatient visit 6 months after surgery.

\section{Statistical Analysis}

The anesthesiologist and performing surgeon were required to complete a questionnaire on perioperative handling, intraoperative effects, aneurysm preparation, and clip application. Data were collected prospectively into an SPSS database (IBM Corp.). An unpaired t-test was used for parametric statistics. Categorical variables were analyzed in contingency tables using the Fisher exact test. Results with a $\mathrm{p}$ value $\leq 0.05$ were considered statistically significant. All calculations were made with standard commercial software (IBM SPSS).

\section{Trial Registration}

This prospective noninterventional study was approved by the local ethics committee of Goethe University Hospital Frankfurt and registered with the ClinicalTrials.gov database (http://clinicaltrials.gov); its registration no. is NCT02766972.

\section{Results}

During the 31-month study period, 174 patients with UIAs were treated. Twenty patients (11\%) with complex UIAs were included in this trial (Tables 1 and 2). No aneurysm ruptured during treadmill ECG, TTE, or RVP. The mean age of the patients was 51.6 years (range 28-66 years), and $75 \%$ were female.

\section{Application of RVP}

Aneurysm preparation and clip application was first attempted without the use of RVP. Intraoperative RVP was not required for safe clip application in 3 patients (15\%). In 1 patient (5\%), the aneurysm was not treated at all because it was predominantly a fusiform aneurysm and heavily calcified, as detected during intraoperative inspection. Therefore, in $16(80 \%)$ of 20 patients RVP was used intraoperatively. In all patients (100\%) the pacemaker electrodes were placed in the anesthesiology preparation room (Fig. 3) and tested for a few seconds. Probe placement and testing required 5-10 minutes. In 1 patient an ultrasound was needed to verify probe placement; thus this patient spent 20 minutes more in the preparation room than a patient without RVP.

Therefore, 24 times RVP was successfully applied in $15(94 \%)$ of 16 patients (Fig. 3, Table 2). Most patients re- 
TABLE 2. Parameters of patients with successful RVP

\begin{tabular}{ccccccc}
\hline $\begin{array}{c}\text { Case } \\
\text { No. }\end{array}$ & Episodes & $\begin{array}{c}\text { Duration } \\
(\text { secs })\end{array}$ & $\begin{array}{c}\text { MAP } \\
(\mathrm{mm} \mathrm{Hg})\end{array}$ & $\begin{array}{c}\text { HR } \\
(\mathrm{bpm})\end{array}$ & Benefit & Comment \\
\hline 1 & 4 & $60,55,70,30$ & $40-50$ & 180 & AP, CA & \\
\hline 2 & 1 & 55 & 50 & 200 & AP, CA & \\
\hline 3 & 2 & 40,50 & 50 & 200 & CA & Electrical cardioversion due to arrhythmia intraoperatively after 2nd RVP \\
\hline 5 & 1 & 120 & 50 & 180 & AP, CA & Ventricular fibrillation, approximately 50 chest compressions required \\
\hline 7 & 2 & 37,34 & $43-50$ & 170 & CA & \\
\hline 9 & 1 & 40 & 53 & 150 & CA & \\
\hline 11 & 1 & 67 & 54 & 150 & CA & \\
\hline 12 & 1 & 67 & 49 & 150 & AP, CA & \\
\hline 13 & 2 & 50,45 & 50 & 140 & CA & \\
\hline 16 & 3 & $20,70,75$ & $40-50$ & $133-152$ & CA & Additional temporary clipping for clip removal due to clip dislocation \\
\hline 17 & 1 & 70 & 50 & 190 & CA & \\
\hline 18 & 2 & 55,72 & $36-43$ & 210 & CA & \\
\hline 19 & 1 & $105^{*}$ & 45 & 185 & AP, CA & Second RVP failed due to dislocation of pacemaker electrode; therefore, \\
\hline 20 & 1 & 70 & 48 & 186 & AP, CA & additional temporary clipping required \\
\hline
\end{tabular}

$\mathrm{AP}=$ aneurysm preparation and dissection; $\mathrm{CA}=$ clip application; $\mathrm{HR}=$ heart rate.

The effect of RVP was classified as "excellent" in all technical successful maneuvers.

* Second RVP failed due to dislocation of the pacemaker electrode.

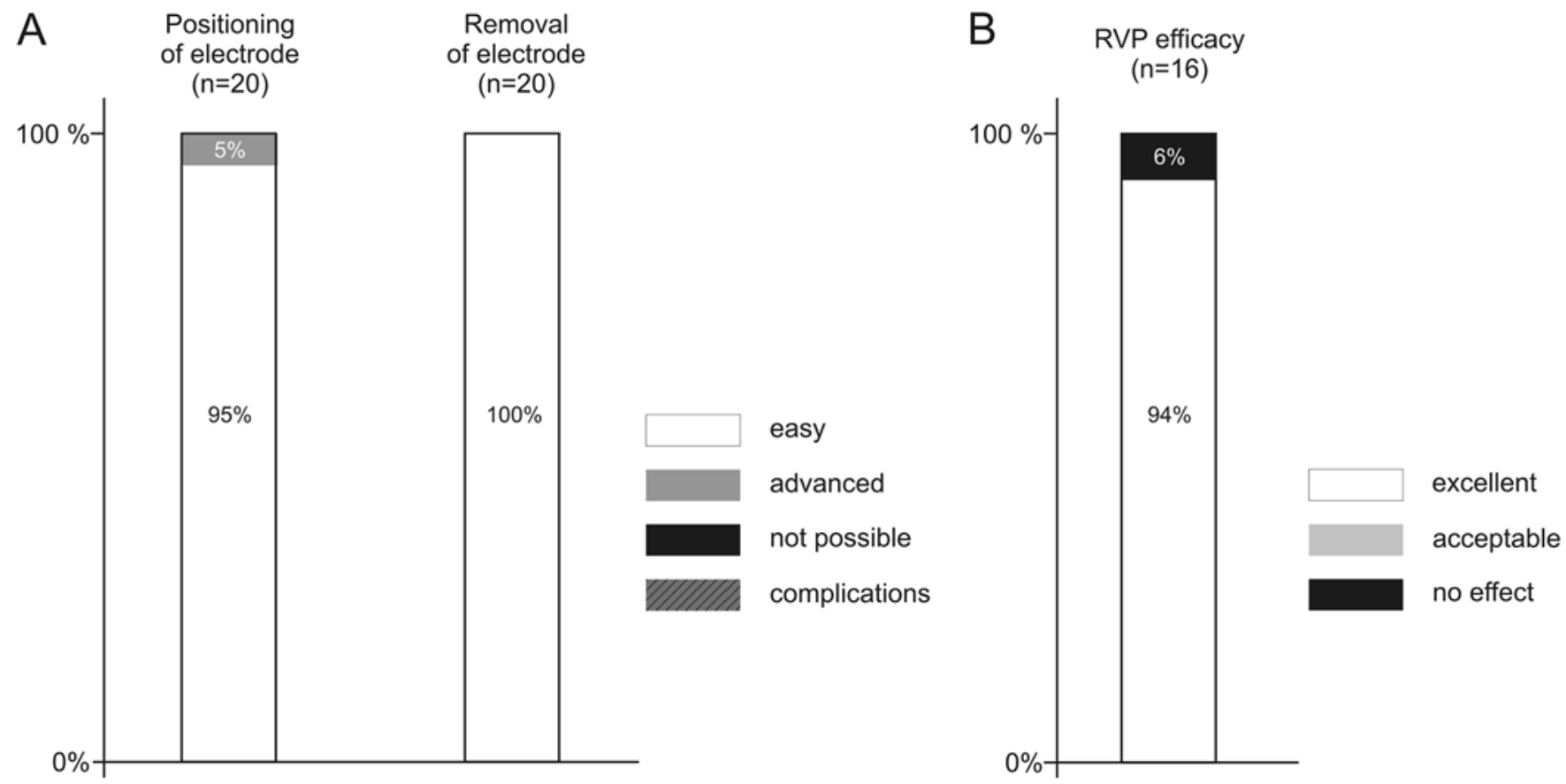

FIG. 3. Graph of the survey results showing the ratings of the anesthesiologist (A) and neurosurgeon (B). Positioning of electrodes was easy in 19 of 20 patients (left column). For 1 patient a transesophageal echocardiogram was required. No complications occurred. Electrode removal was easy and no complications occurred (center column). In 15 of 16 patients the effect of RVP was evaluated as excellent (right column). However, due to pacemaker dislocation in 1 patient, RVP failed (Case 14), and in another patient, after an initial RVP with excellent effect, the second RVP did not work (Case 18). However, a repositioning is usually easy, and after adopting the internal guidelines and allowing this repositioning in Case 20, the electrodes were corrected and the RVP had an excellent effect (MAP $35 \mathrm{~mm} \mathrm{Hg}$ ). 
TABLE 3. Cardiovascular blood levels in patients with and without intraoperative use of RVP

\begin{tabular}{|c|c|c|c|c|c|c|}
\hline \multirow[b]{2}{*}{ Variable } & \multicolumn{3}{|c|}{ Postop } & \multicolumn{3}{|c|}{ Day 1 After Op } \\
\hline & Troponin T (pg/ml) & CK (U/L) & CK-MB (U/L) & Troponin T (pg/ml) & CK (U/L) & CK-MB (U/L) \\
\hline Normal blood values & $<50$ (gray area $14-50$ ) & $<190$ & $<24$ & $<50$ (gray area 14-50) & $<190$ & $<24$ \\
\hline w/ RVP & 6 & 122 & 13 & 5 & 278 & 17 \\
\hline w/o RVP & 14 & 160 & 16 & 7 & 329 & 20 \\
\hline p Value* & 0.21 (NS) & 0.15 (NS) & 0.26 (NS) & 0.52 (NS) & 0.12 (NS) & 0.34 (NS) \\
\hline
\end{tabular}

NS = nonsignificant.

Mean values are presented. In the RVP group the levels were not significantly lower compared with the 4 patients not requiring RVP.

* Mann-Whitney U-test.

quired only 1 pacing, but RVP was used up to 4 times in 1 patient (Case 1, Table 2). During a mean duration of 60 \pm 25 seconds of RVP with a mean heart rate of $173 \pm 23$ bpm (range 150-210 bpm), a reduction of the MAP (range $35-55 \mathrm{~mm} \mathrm{Hg}$ ) was achieved. This led to a significant reduction of the wall tension of the aneurysm and thus facilitated the preparation of the aneurysm and clip application or parent-vessel clip reconstruction with aneurysm occlusion (Fig. 3, Table 3).

In $3(19 \%)$ of 16 patients RVP failed due to pacemaker lead dislocation. In 1 of these patients (Case 14), despite the use of other techniques (induced hypotension and temporary clipping), the complex posterior communicating artery (PCoA) aneurysm (14 mm, neck incorporating PCoA origin and posterior wall of the internal carotid artery [ICA]) could not be clipped safely without the use of RVP so the procedure was terminated without securing the aneurysm. This aneurysm was treated subsequently by placement of a flow diverter because conventional coiling was not possible due to the configuration of the aneurysm. In another patient (Case 18) the first RVP was successful as preparation of both $A_{2}$ segments (which were very adherent to the aneurysm sac) was performed easily and clip application was performed. However, ICG showed a significant remnant, so a second RVP was tried but failed due to pacemaker electrode dislocation. Therefore, additional temporary clipping was used instead, but flow reduction was not as sufficient as under RVP. This led to an incomplete clipping of the aneurysm and finally a remnant on postoperative DSA. However, if the pacemaker electrodes were positioned correctly in the preparation room, a repositioning was not that difficult. Thus, we changed our internal guidelines and allowed intraoperative repositioning. In the third patient (Case 20) the first RVP failed due to dislocation of the pacemaker electrode. Repositioning required less than 20 seconds, and the complex middle cerebral artery (MCA) trifurcation aneurysm could be clipped successfully. During RVP, no changes in the intraoperatively measured SSEPs, MEPs, or EEGs were detected.

\section{Aneurysm Characteristics}

The mean size of the aneurysms was $11.1 \pm 5.5 \mathrm{~mm}$ (range 6-30 mm). Seven aneurysms were large $(\geq 10 \mathrm{~mm}$ ) and 1 was giant $(30 \mathrm{~mm})$. The majority of aneurysms had a wide neck and/or were dysplastic, and at least 1 origin of the branching vessels was incorporated (Figs. 4-6).
In 15 (94\%) of 16 patients, RVP was applied intraoperatively to secure the aneurysm. Mean aneurysm size was $11.6 \pm 6.2 \mathrm{~mm}$, with 6 aneurysms $\geq 10 \mathrm{~mm}$. The neck was wide in nearly all and calcified in most of these aneurysms, which made clip application more difficult and sometimes prevented the closure of the clip blades. Dislocation of the clip downward to the parent vessel was noted in some of the aneurysms when a first attempt at clipping was performed without using RVP. This was due to calcifications in the region of the neck or the increased tension of the aneurysm wall. In 1 of these aneurysms, an endovascular approach was aborted without success prior to clip placement (Case 12). In most of the aneurysms the branching vessels were at least partly incorporated at the origin of the aneurysm. Two aneurysms originated at the paraclinoid ICA, where intradural proximal control is difficult to achieve and visualization of the clip blades during closure is limited.

\section{Cardiovascular Events}

In most patients, no cardiovascular events were recorded during or after the procedure. The serum levels of cardiovascular markers (troponin T, creatine kinase [CK], and CK-MB) were within normal range or slightly elevated. However, the levels of patients with RVP were lower than the levels without use of RVP intraoperatively (Table 3 ).

In $1(6 \%)$ of the 16 patients (Case 3) electrical cardioversion was applied after the second episode of RVP intraoperatively due to supraventricular tachycardia. The fourth patient who underwent RVP intraoperatively (Case 5) developed a pulseless electrical activity with ventricular fibrillation after RVP due to extensive stimulation (120 seconds) requiring approximately 50 chest compressions before spontaneous recovery. Both patients made a full recovery. No other arrhythmias or ischemic abnormalities were documented during 24 hours of continuous ECG monitoring after the procedure. However, after adopting our internal guidelines (RVP autonomously stopped by the anesthesiologist after 100 seconds), no further pulseless electrical activity with ventricular fibrillation occurred.

No complications associated with placement of the pacing electrodes occurred, such as cardiac perforation or cardiac tamponade. One patient (Case 8) suffered from myocardial infarction 10 months after the procedure. RVP was not applied intraoperatively in this patient and the preoperative diagnostics did not display any abnormalities. 

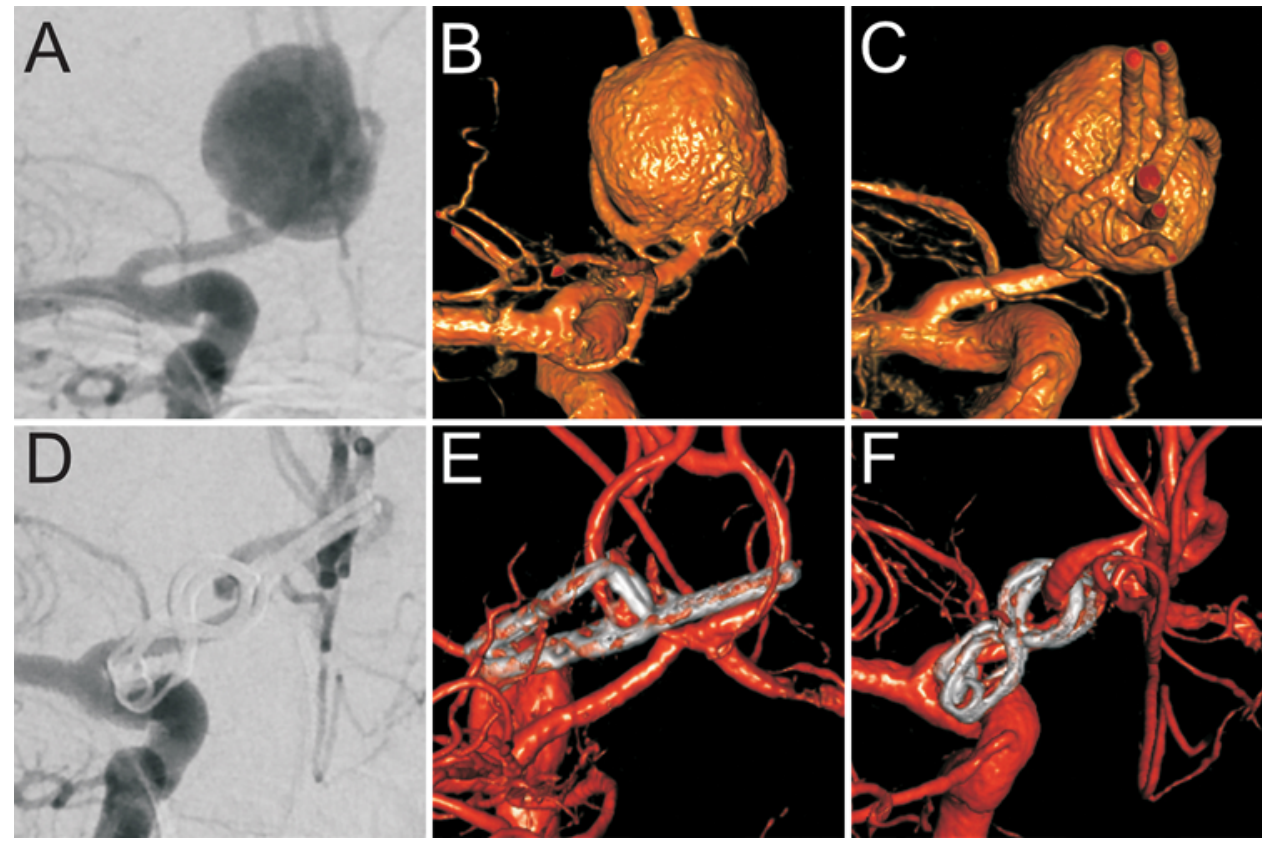

FIG. 4. Case 1. Angiograms ( $A$ and $D)$ and $3 D$ DS angiograms ( $B, C, E$, and $F)$ showing a complex, large (18-mm), wide-neck anterior communicating artery $(\mathrm{ACo} A)$ aneurysm $(\mathbf{A}-\mathbf{C})$ in a 66-year-old man. The aneurysm was adjacent to the $\mathrm{A}_{2}$ segments (B and C). After 4 episodes of RVP $(60,55,70$, and 30 seconds; heart rate approximately $180 \mathrm{bpm}$ ) with a MAP reduction to 40 $\mathrm{mm} \mathrm{Hg}$, the aneurysm was successfully clipped (D-F). Figure is available in color online only.

\section{Outcome}

Outcome 6 months after aneurysm occlusion was favorable in all patients $(\mathrm{n}=19 ; \mathrm{mRS}$ score $0-2)$. Only 1 patient had an mRS score of 2 (pre- and postoperatively), but this was related to a previous SAH from a different aneurysm. One patient (treated in March 2016) had an mRS score of 1 at discharge.

\section{Discussion}

The treatment of complex and large UIAs remains challenging to date. Complete circumferential exposure of the aneurysm, branches, and perforators is required prior to safe and effective clip application. Yet, aneurysm dissection and visualization of major branches or perforators incorporated into the aneurysm base can be very demanding. Despite the development of various supplementary techniques to facilitate neurosurgical clip reconstruction in the past, such as induced hypotension, suction decompression, temporary clipping, transient cardiac standstill, or bypass surgery, every method still has its specific drawbacks. Also, the application of advanced techniques, which usually carry an additional risk for the patient, has to be weighed against the significant risk of intraoperative aneurysm rupture in these complex aneurysms.

In this study, we prospectively assessed the safety and efficacy of RVP in microsurgical clip reconstruction of complex UIAs in an interdisciplinary setting. We found that RVP is a very efficient technique that facilitates clip reconstruction with a low risk for the patient. RVP works mainly by softening of the aneurysm sac, even in very large or complex aneurysms as well as in calcified aneurysms, which then significantly improves the aneurysm mobiliza- tion and allows better visualization of the aneurysm's neck and branching vessels.

RVP decreases cardiac output and MAP by reducing the stroke volume by desynchronization of the atrial and ventricular action, ventricular dyskinesis, and a shorter diastolic filling time. ${ }^{44}$ Onset and duration can be individually adapted to the needs of the intervention., ${ }^{9,4}$ After its first description in neurosurgery in the 1960s, this technique was abandoned in the 1970s and almost forgotten until 1 small series and 3 case reports reported its successful use in cerebrovascular surgery. ${ }^{21,30,38,45}$

\section{Patient Safety}

In this series, two cardiovascular events were noted in relation to RVP: 1 patient suffered from ventricular fibrillation while another developed atrial fibrillation, which both resolved after adequate intraoperative measures without sequelae. We suspect that some degree of hypovolemia leading to a reduction of myocardial perfusion during RVP might have contributed to the development of ventricular fibrillation in the affected patient. Second, the heart rate used for RVP might have been too high in this patient. After changing our guidelines with a focus on avoidance of hypovolemia and a stepwise increase of the pacing velocity until a sufficient effect was noted, we did not observe a similar event.

Both events are known complications of RVP, which are reported to be between $0 \%$ and $3 \%$. Tachyarrhythmias are the most common complications, which are usually reversed with defibrillation or cardioversion..$^{21,44}$ This risk is highest in patients with aortic stenosis, severe left ventricular dysfunction, or coronary heart disease, which therefore 

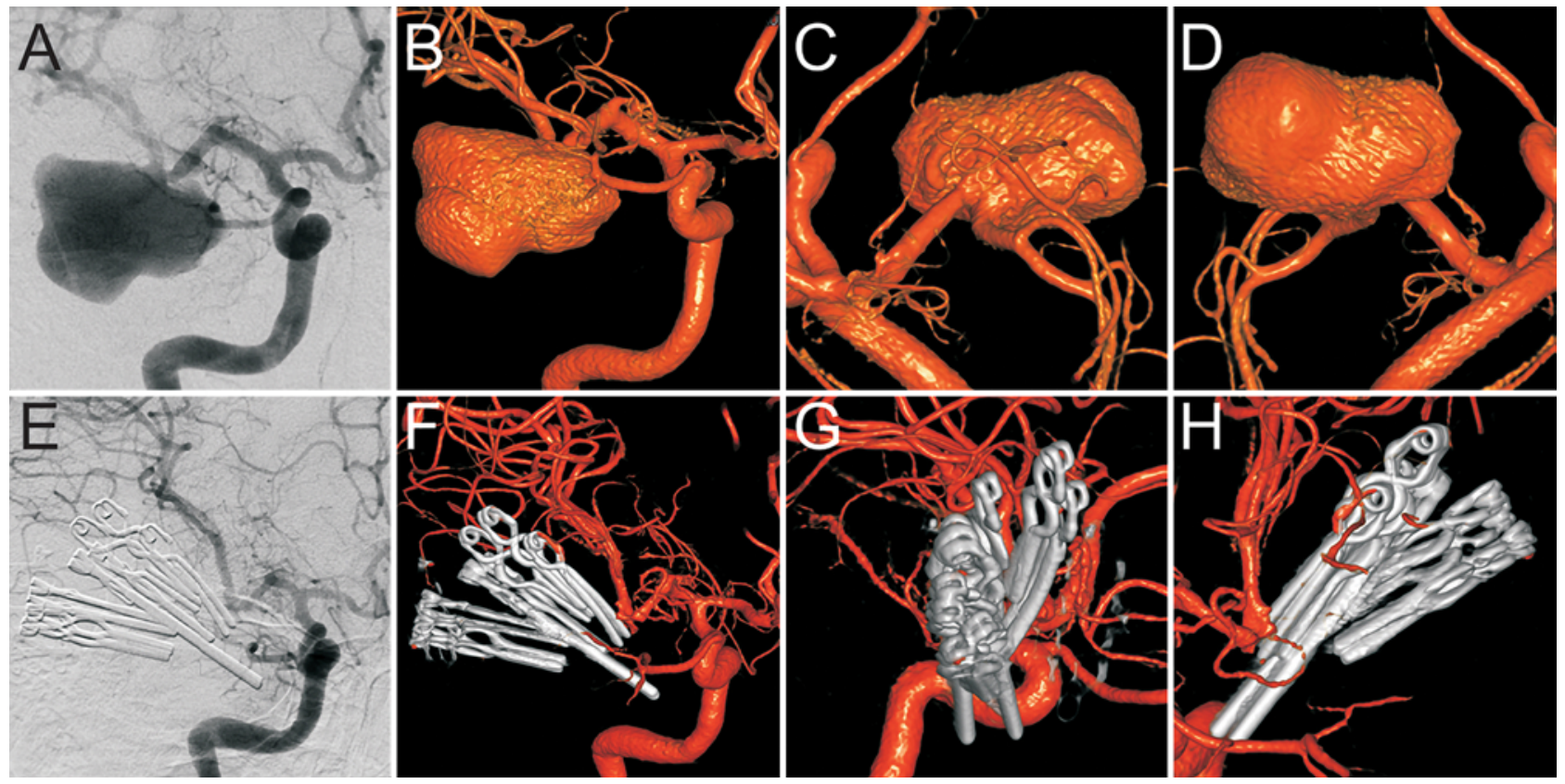

FIG. 5. Case 15. Angiograms ( $A$ and $E$ ) and $3 D$ DS angiograms (B-D, F-H) demonstrating a complex giant (30-mm) MCA aneurysm with a dysplastic calcified neck, including $M_{2}$ origins (A-D). During 3 episodes of RVP $(20,70$ and 75 seconds; heart rate approximately $150 \mathrm{bpm}, \mathrm{MAP} 40-50 \mathrm{~mm} \mathrm{Hg}$ ), 9 clips could be inserted to reconstruct the anatomy (E-H). Figure is available in color online only.

have to be excluded before starting RVP..$^{11,44}$ Therefore, it appears reasonable to limit the frequency and duration of $\mathrm{RVP}$ as much as possible and to ensure adequate recovery of left ventricular systolic function and MAP prior to further pacing..$^{4}$

Interestingly, patients who underwent RVP did not have the highest levels of heart enzymes postoperatively, and no signs of cardiac infarctions. A neurological deficit did not occur. Interestingly, in patients with successful clip placement with RVP, the levels were lower than in patients with non-RVP clipping.

A fundamental rule in our experience is that the anesthesiologist has to monitor the duration of RVP application and inform the surgeon at 30-, 60-, and 90-second time periods so that RVP can be terminated after 100 seconds. The MAP should not regularly be below $40 \mathrm{~mm} \mathrm{Hg}$.

No other arrhythmias or ischemic abnormalities were documented during 24 hours of continuous ECG monitoring after the procedure. However, after adopting our internal guidelines (focus on avoidance of hypovolemia and a stepwise increase of the pacing velocity; RVP terminated after 100 seconds), no further pulseless electrical activity or arrhythmias occurred. No complications associated with placement of the pacing electrodes occurred, such as cardiac perforation or cardiac tamponade (Fig. 3).

One of the major benefits of RVP, especially compared with temporary clipping, is the residual flow (of heart, body, and brain) resulting in constant and slow cerebral perfusion. In contrast to temporary clipping, the IOM identified no alterations of EEGs, SSEPs, or MEPs, which is supported by the data of Argiriadou et al., who showed that RVP did not affect cerebral oxygenation. ${ }^{2}$ The outcome of the patients in the present series was excellent, especially considering the complexity of the treated aneurysms.

\section{Simplicity of RVP}

After discussion of the procedure by our ethics board, we had to use high standards to include the patient participants. Nonetheless, an exercise-based ECG and TTE are currently standard procedures. The implantation of the catheter was performed by an anesthesiologist. The survey showed that a transesophageal echocardiogram was required for preoperative implantation into the right ventricle in only 1 patient (5\%; Fig. 3). However, in 3 (19\%) of 16 patients a dislocation occurred. In 2 patients prior to and in 1 patient after the first use of RVP, electrode dislocation occurred. After changing the internal guidelines (and allowing repositioning after skin incision), in 1 patient (Case 20) the catheter was repositioned very easily (1-2 mm inside) and fast (less than 20 seconds). Therefore, we documented a translocation of the pacemaker lead in $15 \%$. RVP is an elegant technique, and (as requested by the surgeon) the anesthesiologist has a device with on/off demand for a tailored induction of hypotension (i.e., heart rate increase) with residual cerebral and heart perfusion.

\section{Efficacy of Flow Reduction and Improvement of Clip Application}

In every patient with successful RVP $(n=15)$ the operating surgeon (V.S.) rated the efficacy of flow arrest as excellent (100\%; Fig. 3). However, in 1 (6\%) of 16 patients RVP failed due to lead dislocation. One of the most important advantages is the control of RVP, specifically flow reduction. After visualization of the aneurysm, the surgeon 

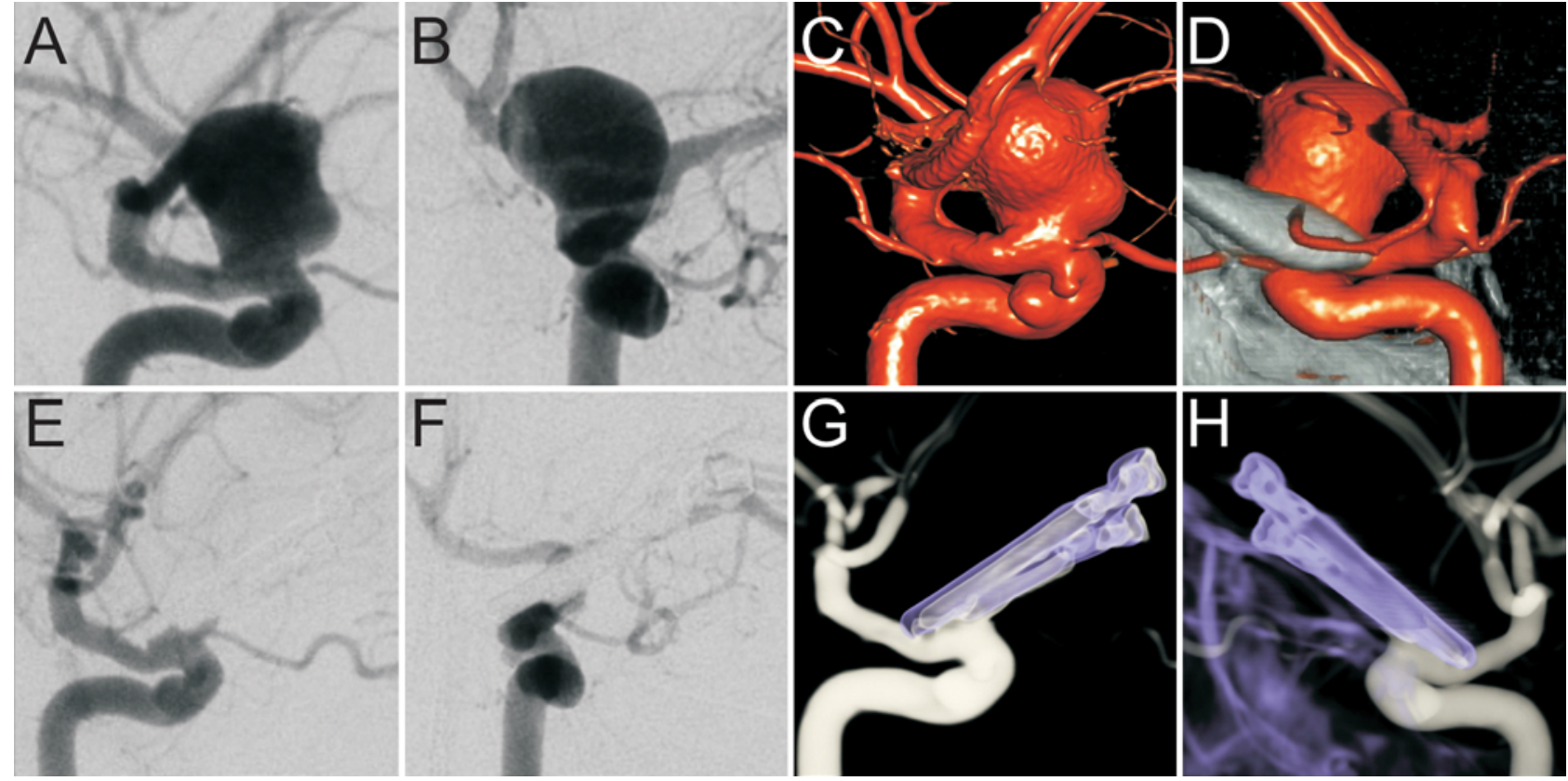

FIG. 6. Case 16. Large (15-mm) paraclinoid ICA aneurysm. For this large paraclinoid aneurysm (A-D) an exclusively intradural exposure with optic nerve unroofing and tailored clinoidectomy was used as previously described..$^{39}$ Instead of suction decompression, only 1 episode of RVP was required (70 seconds; heart rate $190 \mathrm{bpm}$, MAP $50 \mathrm{~mm} \mathrm{Hg}$ ) to treat the aneurysm successfully $(\mathrm{E}-\mathrm{H})$. Figure is available in color online only.

gives a command to be prepared for clip application or dissection of perforators, and the anesthesiologist begins RVP. Seconds later the aneurysm becomes slack and soft. Subsequently, clip application is much easier because of the reduced flow (Video 1).

VIDEO 1. Clip showing our first patient treated using RVP (ACoA aneurysm, Case 1). Copyright Juergen Konczalla. Published with permission. Click here to view.

Most often, RVP was used directly for the application of the clips, which allowed better control and visualization of the closure of the blades. The aneurysm sac became significantly softer by reduced wall tension during RVP in all cases $(100 \%)$, which facilitated its mobilization and manipulation. Thus, dissection of adhering vessels to the aneurysm neck, and circumferential visualization of the neck and the branching or perforating vessels, was improved.

\section{Why RVP and Not Adenosine?}

The complication rate after RVP, which has to be weighed against the considerable risk of spontaneous rupture of the aneurysms treated in this series, appears to be comparable to or lower than that reported after administration of adenosine in retrospective studies (Table 4), ${ }^{4,5,22}$ Adenosine is easy available and usable, but it is not predictable, if the cardiac arrest persists (up to $20 \%$ of the patients had no effect in a prospective trial $\left.{ }^{11}\right)$ and depending on how long (most often 10-30 seconds); $3,5,18,25$ with RVP the anesthesiologist has a device with on/off demand for a tailored induction of hypotension. Also, after adenosine application, hypotension persists longer (5-10 minutes) than after RVP, requiring vasoactive agents in some patients. ${ }^{1,18,26}$ RVP data exist showing that cerebral oxy- genation is not affected, ${ }^{2}$ whereas induced hypotension by adenosine, nitroglycerin, nitroprusside, isoflurane, or others should be as short as possible, as we know from studies that it is associated with reduction of cerebral oxygenation and associated with permanent deficits and stroke depending on duration and extent of hypotension. ${ }^{16,19,24,33,40,47} \mathrm{In}$ contrast to RVP vasoactive agents, sometimes several are required after adenosine or induced hypotension.

However, the cardiac risks of adenosine and RVP are similar (arrhythmias), and elective patients should have the same preoperative cardiac checkup, especially in a prospective trial. Finally, adenosine has a high side-effect profile, whereas RVP is established from transcatheter aortic valve implantation and is very safe. ${ }^{9,11,44}$ In contrast to adenosine, RVP cannot be administrated spontaneously in the case of intraoperative aneurysmal rupture ${ }^{4}$ as the pacemaker electrode needs to be in place beforehand. However, if the electrode was placed in the preparation room, RVP can be used in patients with SAH as well as

\section{TABLE 4. Adverse events}

\begin{tabular}{lcc}
\hline \multicolumn{1}{c}{ Event } & Current RVP Series & Adenosine $3,4,18,22,25$ \\
\hline Atrial fibrillation & $6 \%$ & $5 \%-9 \%$ \\
\hline Elevated troponin levels & $0 \%$ & $8 \%-12 \%$ \\
\hline (Transient) arrhythmias & $6 \%$ & $8 \%-13 \%$ \\
\hline Closed chest compressions & $6 \%$ & $0 \%-4 \%$ \\
\hline Prolonged hypotension & $0 \%$ & Up to $60 \%$ \\
\hline Postop neurological deficits & $0 \%$ & $0 \%-12.5 \%$ \\
\hline
\end{tabular}


during rupture to control bleeding. ${ }^{38}$ In addition, a preoperative cardiac workup is highly recommended. During screening 1 patient was excluded because the treadmill ECG showed ST elevations and the patient required cardiac stents.

However, with adenosine, atrial fibrillation was reported in 5\%-9\%, elevated troponin levels without evidence of cardiac dysfunction in 8\%-12\%, transient arrhythmias occurred in $8 \%-13 \%$, closed chest compressions due to prolonged hypotension were required in $4 \%$, and postoperative neurological deficits occurred in as many as $12.5 \%$ in a retrospective trial. ${ }^{3,4,18,25}$ Despite the required chest compression (6\%), in our prospective series RVP had better values than using adenosine (Table 4). However, we used adenosine earlier and changed to RVP. We prefer RVP, especially due to the good control of flow reduction (by time and effect) in contrast to the unpredictable adenosine and the requirement of vasoactive agents. Additionally, in these complex UIAs with clip reconstruction, the majority of RVP procedures required a duration of $\geq 40 \mathrm{sec}-$ onds (Figs. 4 and 5), which is typically not possible with adenosine. Nonetheless, both techniques are eligible for aneurysm surgery, and data comparing both techniques are still missing.

\section{Why RVP and Not Temporary Clip Placement or Revascularization?}

Compared with temporary clip placement, which also leads to a reduction of aneurysm wall tension, there is residual perfusion in the dependent vessel territory under RVP. In contrast to temporary clipping, where several minutes of flow arrest are usually achieved, the duration achieved by RVP is shorter. ${ }^{17}$ Also in contrast to temporary clipping, the IOM identified no alterations of EEGs, SSEPs, and MEPs, and cerebral oxygenation was also not affected. ${ }^{2}$

Additionally, temporary clipping is associated with the risk of vessel wall injury or thromboembolic complications. ${ }^{5,13}$ Especially in the presence of vessel-wall sclerosis or a prolonged temporary clipping interval, postoperative ischemic deficits may occur. ${ }^{17}$ Sometimes the temporary clip itself might limit the application of the permanent clip, especially in narrow surgical corridors. ${ }^{29}$ For paraclinoid aneurysms (Fig. 6) we used carotid artery occlusion, but similar to intracranial temporary clipping of the carotid artery, in the cervical ICA thrombosis can also occur. ${ }^{5}$ However, we also used additional temporary clipping in some cases. As mentioned previously, in 1 patient the first RVP was successful and preparation of both $A_{2}$ segments (which were very adherent to the aneurysm sac) was relatively easily, whereas temporary clipping was not as sufficient as RVP. Therefore, temporary clipping is an adequate technique but, depending on collateral circulation, sometimes not as effective for general blood flow reduction due to RVP.

We believe that clip reconstruction of the aneurysm and preservation of the patient's vasculature is superior to any revascularization procedure. We therefore spare no effort to achieve this goal. In contrast to RVP (Table 4), revascularization procedures showed higher risks of periprocedural ischemia (up to $29 \%)^{6}$ for complex cerebral aneurysms, which is often associated with a significant morbidity rate. ${ }^{41}$ However, with RVP or adenosine a few aneurysms cannot be reconstructed, and for these cases revascularization techniques may be an alternative.

\section{Limitations of the Study}

Besides the limited number of patients, which is related to the complex nature of the aneurysms selected for RVP application at our institution, there are other limitations to this study. We did not compare the safety and efficacy of RVP to other methods of transient cardiac standstill such as adenosine injection. Furthermore, there is no control group included in this series, but this can hardly be achieved due to the complexity of each individual aneurysm.

At present, after we have used RVP successfully in 15 of 20 patients more than 20 times, and after adjusting the guidelines, we feel confident that we have identified a safe corridor for RVP use: 1) after insertion of electrode, test RVP (with a starting heart rate of $150 \mathrm{bpm}$ ) to find a heart rate for a MAP of approximately $50 \mathrm{~mm} \mathrm{Hg}$; 2) intraoperatively reposition the pacemaker lead if it dislocates; and 3) monitor the duration of RVP application by an anesthesiologist and inform the surgeon at 30-, 60-, and 90-second time periods. RVP should be terminated after 100 seconds during 1 pacing.

\section{Conclusions}

RVP is an elegant technique that significantly improves clip reconstruction in complex UIAs. The safety of the procedure is good. Yet, because this technique requires extensive preoperative cardiological workup of the patient and an experienced neurosurgical and neuroanesthesiology team with much cerebrovascular expertise, actually it remains reserved for selected elective cases and highly specialized centers. After having accumulated experience with RVP we believe it should be further evaluated in a multicenter trial.

\section{Acknowledgments}

We thank Marina Heibel and Anne Sicking for their excellent technical support.

\section{References}

1. Andrade-Barazarte H, Luostarinen T, Goehre F, Kivelev J, Jahromi BR, Ludtka C, et al: Transient cardiac arrest induced by adenosine: a tool for contralateral clipping of internal carotid artery-ophthalmic segment aneurysms. World Neurosurg 84:1933-1940, 2015

2. Argiriadou H, Anastasiadis K, Karapanagiotidis G, Papakonstantinou C: Subclinical decline in cerebral oxymetry saturation during rapid pacing in transfemoral aortic valve replacement. Ann Thorac Surg 90:1023, 2010

3. Bebawy JF, Gupta DK, Bendok BR, Hemmer LB, Zeeni C, Avram MJ, et al: Adenosine-induced flow arrest to facilitate intracranial aneurysm clip ligation: dose-response data and safety profile. Anesth Analg 110:1406-1411, 2010

4. Bendok BR, Gupta DK, Rahme RJ, Eddleman CS, Adel JG, Sherma AK, et al: Adenosine for temporary flow arrest during intracranial aneurysm surgery: a single-center retrospective review. Neurosurgery 69:815-821, 2011

5. Benech CA, Perez R, Faccani G, Trompeo AC, Cavallo S, 
Beninati S, et al: Adenosine-induced cardiac arrest in complex cerebral aneurysms surgery: an Italian single-center experience. J Neurosurg Sci 58:87-94, 2014

6. Brilstra EH, Rinkel GJE, Klijn CJM, van der Zwan A, Algra A, Lo RTH, et al: Excimer laser-assisted bypass in aneurysm treatment: short-term outcomes. J Neurosurg 97:1029-1035, 2002

7. Chalouhi N, Polifka A, Daou B, Kung D, Barros G, Tjoumakaris S, et al: In-pipeline stenosis: incidence, predictors, and clinical outcomes. Neurosurgery 77:875-879, 2015

8. Cognard C, Januel AC: Remnants and recurrences after the use of the WEB intrasaccular device in large-neck bifurcation aneurysms. Neurosurgery 76:522-530, 2015

9. Daehnert I, Rotzsch C, Wiener M, Schneider P: Rapid right ventricular pacing is an alternative to adenosine in catheter interventional procedures for congenital heart disease. Heart 90:1047-1050, 2004

10. Darsaut TE, Darsaut NM, Chang SD, Silverberg GD, Shuer LM, Tian L, et al: Predictors of clinical and angiographic outcome after surgical or endovascular therapy of very large and giant intracranial aneurysms. Neurosurgery 68:903915, 2011

11. Davidavicius G, Chieffo A, Shannon J, Arioli F, Ielasi A, Mussardo M, et al: A high dose of adenosine to induce transient asystole for valvuloplasty in patients undergoing transcatheter aortic valve implantation (TAVI): is it a valid alternative to rapid pacing? A prospective pilot study. J Invasive Cardiol 23:467-471, 2011

12. Eliava SS, Filatov YM, Yakovlev SB, Shekhtman OD, Kheireddin AS, Sazonov IA, et al: Results of microsurgical treatment of large and giant ICA aneurysms using the retrograde suction decompression (RSD) technique: series of 92 patients. World Neurosurg 73:683-687, 2010

13. Ferch R, Pasqualin A, Pinna G, Chioffi F, Bricolo A: Temporary arterial occlusion in the repair of ruptured intracranial aneurysms: an analysis of risk factors for stroke. J Neurosurg 97:836-842, 2002

14. Fulkerson DH, Horner TG, Payner TD, Leipzig TJ, Scott JA, Denardo AJ, et al: Endovascular retrograde suction decompression as an adjunct to surgical treatment of ophthalmic aneurysms: analysis of risks and clinical outcomes. Neurosurgery 64 (3 Suppl):ons107-ons112, 2009

15. Gascou G, Lobotesis K, Brunel H, Machi P, Riquelme C, Eker O, et al: Extra-aneurysmal flow modification following pipeline embolization device implantation: focus on regional branches, perforators, and the parent vessel. AJNR Am J Neuroradiol 36:725-731, 2015

16. Giannotta SL, Oppenheimer JH, Levy ML, Zelman V: Management of intraoperative rupture of aneurysm without hypotension. Neurosurgery 28:531-536, 1991

17. Griessenauer CJ, Poston TL, Shoja MM, Mortazavi MM, Falola M, Tubbs RS, et al: The impact of temporary artery occlusion during intracranial aneurysm surgery on long-term clinical outcome: Part II. The patient who undergoes elective clipping. World Neurosurg 82:402-408, 2014

18. Guinn NR, McDonagh DL, Borel CO, Wright DR, Zomorodi AR, Powers CJ, et al: Adenosine-induced transient asystole for intracranial aneurysm surgery: a retrospective review. J Neurosurg Anesthesiol 23:35-40, 2011

19. Hitchcock ER, Tsementzis SA, Dow AA: Short- and longterm prognosis of patients with a subarachnoid haemorrhage in relation to intra-operative period of hypotension. Acta Neurochir (Wien) 70:235-242, 1984

20. Kalani MYS, Ramey W, Albuquerque FC, McDougall CG, Nakaji P, Zabramski JM, et al: Revascularization and aneurysm surgery: techniques, indications, and outcomes in the endovascular era. Neurosurgery 74:482-498, 2014

21. Khan SA, Berger M, Agrawal A, Huang M, Karikari I, Nimjee SM, et al: Rapid ventricular pacing assisted hypotension in the management of sudden intraoperative hemorrhage during cerebral aneurysm clipping. Asian J Neurosurg 9:33-35, 2014

22. Khan SA, McDonagh DL, Adogwa O, Gokhale S, Toche UN, Verla T, et al: Perioperative cardiac complications and 30day mortality in patients undergoing intracranial aneurysmal surgery with adenosine-induced flow arrest: a retrospective comparative study. Neurosurgery 74:267-272, 2014

23. Konczalla J, Platz J, Brawanski N, Güresir E, Lescher S, Senft C, et al: Endovascular and surgical treatment of internal carotid bifurcation aneurysms: comparison of results, outcome, and mid-term follow-up. Neurosurgery 76:540-551, 2015

24. Lagerkranser M, Gordon E, Rudehill A: Cardiovascular effects of sodium nitroprusside in cerebral aneurysm surgery. Acta Anaesthesiol Scand 24:426-432, 1980

25. Lee SH, Kwun BD, Kim JU, Choi JH, Ahn JS, Park W, et al: Adenosine-induced transient asystole during intracranial aneurysm surgery: indications, dosing, efficacy, and risks. Acta Neurochir (Wien) 157:1879-1886, 2015

26. Luostarinen T, Takala RSK, Niemi TT, Katila AJ, Niemelä M, Hernesniemi J, et al: Adenosine-induced cardiac arrest during intraoperative cerebral aneurysm rupture. World Neurosurg 73:79-83, e9, 2010

27. Millesi M, Wang WT, Herta J, Bavinzski G, Knosp E, Gruber A: De novo aneurysm formation at the anastomosis site incidentally detected 2 years after single-barrel STA-MCA bypass surgery: case report and review of the literature. $\mathbf{J}$ Neurol Surg A Cent Eur Neurosurg 76:323-327, 2015

28. Morita A, Kirino T, Hashi K, Aoki N, Fukuhara S, Hashimoto N, et al: The natural course of unruptured cerebral aneurysms in a Japanese cohort. N Engl J Med 366:2474-2482, 2012

29. Mukerji N, Cook DJ, Steinberg GK: Temporary clipping for unruptured aneurysms. World Neurosurg 82:309-311, 2014

30. Nimjee SM, Smith TP, Kanter RJ, Ames W, Machovec KA, Grant GA, et al: Rapid ventricular pacing for a basilar artery pseudoaneurysm in a pediatric patient: case report. J Neurosurg Pediatr 15:625-629, 2015

31. Nussbaum ES: Surgical distal outflow occlusion for the treatment of complex intracranial aneurysms: experience with 18 cases. Neurosurgery 11 (Suppl 2):8-16, 2015

32. Pierot L, Klisch J, Liebig T, Gauvrit JY, Leonardi M, Nuzzi NP, et al: WEB-DL endovascular treatment of wide-neck bifurcation aneurysms: long-term results in a European series. AJNR Am J Neuroradiol 36:2314-2319, 2015

33. Plaschke K, Böckler D, Schumacher H, Martin E, Bardenheuer HJ, Bardenheuer HJ: Adenosine-induced cardiac arrest and EEG changes in patients with thoracic aorta endovascular repair. Br J Anaesth 96:310-316, 2006 (Erratum in Br J Anaesth 96:545, 2006)

34. Platz J, Wagner M, Güresir E, You S, Konczalla J, du Mesnil de Rochemont R, et al: Early diffusion-weighted MRI lesions after treatment of unruptured intracranial aneurysms: a prospective study. J Neurosurg 126:1070-1078, 2017

35. Raabe A, Nakaji P, Beck J, Kim LJ, Hsu FPK, Kamerman JD, et al: Prospective evaluation of surgical microscopeintegrated intraoperative near-infrared indocyanine green videoangiography during aneurysm surgery. J Neurosurg 103:982-989, 2005

36. Rangel-Castilla L, Russin JJ, Britz GW, Spetzler RF: Update on transient cardiac standstill in cerebrovascular surgery. Neurosurg Rev 38:595-602, 2015

37. Rovit RL: Operative hypotension for intracranial vascular surgery using pacemaker-induced ventricular tachycardia. J Neurosurg 35:51-58, 1971

38. Saldien V, Menovsky T, Rommens M, Van der Steen G, Van Loock K, Vermeersch G, et al: Rapid ventricular pacing for flow arrest during cerebrovascular surgery: revival of an old 
concept. Neurosurgery 70 (2 Suppl Operative):270-275, 2012

39. Seifert V, Güresir E, Vatter H: Exclusively intradural exposure and clip reconstruction in complex paraclinoid aneurysms. Acta Neurochir (Wien) 153:2103-2109, 2011

40. Stiefel MF, Heuer GG, Abrahams JM, Bloom S, Smith MJ, Maloney-Wilensky E, et al: The effect of nimodipine on cerebral oxygenation in patients with poor-grade subarachnoid hemorrhage. J Neurosurg 101:594-599, 2004

41. Surdell DL, Hage ZA, Eddleman CS, Gupta DK, Bendok BR, Batjer HH: Revascularization for complex intracranial aneurysms. Neurosurg Focus 24(2):E21, 2008

42. Szelényi A, Beck J, Strametz R, Blasel S, Oszvald A, Raabe A, et al: Is the surgical repair of unruptured atherosclerotic aneurysms at a higher risk of intraoperative ischemia? Clin Neurol Neurosurg 113:129-135, 2011

43. Takahashi T, Suzaki N, Tsugane S, Yamauchi K, Takada S: Suction decompression methods for giant internal carotid ophthalmic aneurysms by using revised double lumen balloon catheters. Acta Neurochir Suppl 103:9-10, 2008

44. Webb JG, Pasupati S, Achtem L, Thompson CR: Rapid pacing to facilitate transcatheter prosthetic heart valve implantation. Catheter Cardiovasc Interv 68:199-204, 2006

45. Whiteley JR, Payne R, Rodriguez-Diaz C, Ellegala DB, Reeves ST: Rapid ventricular pacing: a novel technique to decrease cardiac output for giant basilar aneurysm surgery. J Clin Anesth 24:656-658, 2012

46. Wiebers D, Whisnant J, Forbes G, Meissner I, Brown R Jr, Piepgras D, et al: Unruptured intracranial aneurysms-risk of rupture and risks of surgical intervention. N Engl J Med 339:1725-1733, 1998 (Erratum in N Engl J Med 339:17251733, 1998)

47. Zhang X, Hu Q, Liu Z, Huang H, Zhang Q, Dai H: Compari- son between nitroglycerin and remifentanil in acute hypervolemic hemodilution combined with controlled hypotension during intracranial aneurysm surgery. Int J Clin Exp Med 8:19353-19359, 2015

\section{Disclosures}

The authors report no conflict of interest concerning the materials or methods used in this study or the findings specified in this paper.

\section{Author Contributions}

Conception and design: all authors. Acquisition of data: Konczalla, Platz, Fichtlscherer, Mutlak, Strouhal. Analysis and interpretation of data: Konczalla, Platz, Fichtlscherer, Seifert. Drafting the article: Konczalla, Platz. Critically revising the article: Fichtlscherer, Mutlak, Strouhal, Seifert. Reviewed submitted version of manuscript: all authors. Approved the final version of the manuscript on behalf of all authors: Konczalla. Statistical analysis: Konczalla, Platz. Study supervision: Seifert.

\section{Supplemental Information \\ Videos \\ Video 1. https://vimeo.com/212590147.}

\section{Correspondence}

Juergen Konczalla, Department of Neurosurgery, Goethe University Hospital Frankfurt am Main, Schleusenweg 2-16, Frankfurt am Main 60528, Germany. email: j.konczalla@med. uni-frankfurt.de. 